\title{
Evaluation of the novel gene Rabl3 in the regulation of proliferation and motility in human cancer cells
}

\author{
QI LI ${ }^{1,2}$, LI WANG ${ }^{1,2}$, LINGE ZENG ${ }^{1,2}$, YINGMEI ZHANG ${ }^{1,2}$, KANGQI LI ${ }^{1,2}$, \\ PENG JIN ${ }^{1,2}$, BINGNAN SU ${ }^{1}$ and LU WANG ${ }^{1,2}$ \\ ${ }^{1}$ Laboratory of Medical Immunology, School of Basic Medical Science, Peking University Health Science Center, \\ ${ }^{2}$ Peking University Center for Human Disease Genomics, 38 Xueyuan Road, Beijing 100191, P.R. China
}

Received February 25, 2010; Accepted March 30, 2010

DOI: $10.3892 /$ or_00000876

\begin{abstract}
Rab is a large subfamily of small GTPases which play an important role in multiple processes relating to cellular transportation and modulation of the cytoskeleton. Novel gene Rab-like 3 (Rabl3), which we originally reported as a new member belonging to this subfamily, may participate in other processes in human cancer cell lines based on our research. In order to investigate the function of Rabl3 and the basic mechanism which regulates cancer cell survival and motility, we constructed the Rabl3-pcDNA3.1 fusion plasmid, also, the specific siRNA against Rabl3 was used. We detected cellular viability and motility changes in Rabl3 overexpressed or si-Rabl3 transfected cancer cell lines. Overexpression of Rabl3 resulted in the enhancement of cell proliferation, inhibition of apoptosis and paclitaxel resistance in human cancer cell lines. Rabl3 also promoted cell motility activity. Next, we silenced Rabl3 in order to determine its exact physiological function. We found that processes which are associated with tumor formation and metastasis were inhibited. These included an increased incidence of apoptosis, abrogated cellular proliferation and mobility. Furthermore, Western blot analysis revealed that the function of Rabl3 was closely associated with focal adhesion kinase (FAK) phosphorylation, both in HeLa and MDA-MB-231 cell lines at the sites of Tyr 397/576/577. Our results suggested that Rabl3 may be a novel oncogene which regulates the oncological behavior of human cancer cells. As a consequence, Rabl3 can be considered as a novel candidate in the control of tumorigenesis and as a new target for anti-tumor treatment.
\end{abstract}

\section{Introduction}

The Rab subfamily, which is the largest group in the Ras superfamily, comprises small G-proteins which represent the

Correspondence to: Dr Lu Wang, Peking University Center for Human Disease Genomics, 38 Xueyuan Road, Beijing 100191, P.R. China

E-mail: wanglu@bjmu.edu.cn

Key words: Rab, apoptosis, motility, focal adhesion kinase, paclitaxel guanine nucleotide binding proteins (1). More than 60 Rabs have been discovered to exist ubiquitously in various kinds of eukaryocyte organisms, ranging from yeast to mammals (2). The sequences of the Rab subfamily members are highly conserved, with molecular weights ranging between 20 and $26 \mathrm{KD}$. Most of the Rab members can bind to GTP or GDP, functioning as low-molecular weight GTPases and contributing to GDP/GTP conversion.

At the physiological level, they are involved in various kinds of cellular processes or signaling transductions which are crucial for cellular membrane function, cytoskeleton regulation and protein recycling. Therefore, Rabs play key roles in secretion, endo-/exo-cytosis and intra-/inter-trafficking events $(3,4)$. Moreover, the present study provides additional evidence in support of the participation of Rabs in signaling transduction, modification and the crosstalk between signal cascades $(5,6)$.

At the pathological level, the dysfunction of G-proteins results in abnormalities in many central events and can even initiate tumorigenesis, tumor cell invasion or metastasis in some cases (7-9). Rab family members, such as Rab25, Rab20 and Rab2, are highly expressed in cancer cells and have close relationships with the progression and aggressiveness of different types of cancer $(10,11)$. The impact of Rabs on tumor cells, in terms of their modulation of the cytoskeleton and intracellular transportation are well established, but there is limited evidence related to their effects on functional changes in tumor cell viability and motility. As a consequence, efforts to investigate and reveal the multiple biological functions and mechanisms related to these proteins will potentially be of great benefit to clinical practice, and will provide a better understanding of this gene family.

Due to continuing progress in the field of bio-molecular technology and applications developed as a result of the Human Genome Project (HGP), there are increasing numbers of novel Rab genes being identified and cloned. Their structures and functions are distinct from typical Rabs. Some of them are being named Rab-like proteins. Novel gene Rabl3 is one such protein. To date, studies have revealed few functions for this gene, and there are no reports regarding the relationship between Rabl3 and tumorigenesis or tumor cell metastasis.

In the present study, we investigated the functional characterization of Rabl3 as a candidate oncogene and its 
engagement in multiple processes which influence viability, motility and drug resistance in human cancer cells.

\section{Materials and methods}

Reagents and materials. Restricted endonucleases: EcoRI was purchased from Takara (DaLian, China); polyclonal antibody against Rabl3 was obtained from ProteinTech Group Inc. (USA); monoclonal antibody against $\beta$-actin was purchased from Sigma (USA); IRDTMye 800-conjugated secondary IgG antibodies against rabbit or mouse were obtained from LICOR Bioscience (USA); paclitaxel was purchased from Haikou Pharmaceutical Factory Co., Ltd., China; DNA plasmid extraction kit was purchased from Qiagen (USA); FITC-conjugated Annexin V, and propidium iodide (PI) were purchased from Biosea Biotechnology Co., (Beijing, China); RMPI-1640 was purchased from Invitrogen (Carlsbad, CA, USA).

Cell culture and transfection. Human breast cancer cell line MDA-MB-231 and HeLa cell lines were cultured in RPMI1640 medium containing $10 \%$ fetal bovine serum (FBS) and L-glutamine $(2 \mathrm{mM})$. Other cell lines which were used for the analysis of the Rabl3 expression profile were purchased from the American Type Culture Collection (ATCC). All of the cell lines were cultured in a humid incubator at a temperature of $37^{\circ} \mathrm{C}$ containing $5 \% \mathrm{CO}_{2}$. Plasmid transfection of MDA-MB-231 or HeLa cells was performed using an ECM830 electroporation system (BTX, USA) at a voltage of $130 \mathrm{~V}, 20 \mathrm{~ms}$, single pulse. Plasmid $(10 \mu \mathrm{g})$ was used for $1-3 \times 10^{6}$ transfected cells without FBS in 2-mm gap cuvettes.

cDNA cloning and vector construction. The full length of the Rabl3 gene was obtained from the cDNA library of HeLa cell lines through PCR technology with the primer p1: 5'-ATGGCGTCCCTGGATCGGGT-3' and P2: 5'-CATCT A GATGAGCTGTGAAAAACTGCC-3'. The PCR product was ligated into the T-easy vector and then cut off the insert through EcoRI. Subsequently the released Rabl3 insert fragment was subcloned into the pcDNA3.1myc/hisB(-) EcoRI polycloned site, to form the pcDNA3.1myc/his(-)-Rabl3 fusion plasmid. The plasmids mentioned above were confirmed by DNA sequencing.

Rabl3 siRNA synthesis. The Rabl3 specific siRNA was designed and synthesized by a method which targets the sequence of 5'-CAAGAGCAUAUCUACAATT-3'. The mock siRNA was designed with a sequence of 5'-UUCUCCGA ACGUGUCACGUTT-3'. Both siRNAs mentioned above underwent PAGE purification in an environment free of RNase contamination. In accordance with the manufacturer instructions, the siRNA was dissolved at the appropriate concentration $(20 \mu \mathrm{M})$. Dissolved siRNA $(10 \mu \mathrm{l})$ was used for $1-3 \times 10^{6}$ transfected cells in $400 \mu 1$ of FBS free RPMI1640 during the electroporation procedure.

$R T$-PCR analysis. The total mRNA was extracted from the distinct cell lines by TRIzol reagent (Invitrogen, USA) and retranscripted using a iScript ${ }^{\mathrm{TM}}$ c-DNA synthesis kit (Bio-
Red, CA, USA) as indicated in the manufacturer's instructions. Total mRNA $(1 \mu \mathrm{g})$ was used for the RT procedure to synthesize the cDNA library at $42^{\circ} \mathrm{C}$ for $30 \mathrm{~min}$. The cDNA libraries from indicated cell lines were then analyzed using Rabl3 specific primers P3: 5'-TTGGGAGACTCAGGTGT TGGGAAA-3' and P4: 5'-CAGTTGGCACCAAATCCC TGTTGA-3' and the PCR products were separated by agarose electrophoresis. The GAPDH specific primers were also used as the internal control.

Cell apoptosis and proliferation assay. As the key index for apoptosis detection, Annexin V-PI staining was performed to measure phosphatidylserine externalization in MDA-MB-231 cells. Briefly, cells transfected with different plasmids were harvested at the indicated time points. Trypsinized cells were washed twice and resuspended in $200 \mu \mathrm{l}$ of binding buffer (10 mM HEPES, pH 7.4, $140 \mathrm{mM} \mathrm{NaCl}, 1 \mathrm{mM} \mathrm{MgCl} 2,5 \mathrm{mM}$ $\left.\mathrm{KCl}, 2.5 \mathrm{mM} \mathrm{CaCl}_{2}\right)$. FITC-conjugated Annexin V $(10 \mu \mathrm{l})$ was then added to give a final concentration of $0.5 \mu \mathrm{g} / \mathrm{ml}$. The staining sample was incubated at room temperature for 20 min, without light. Subsequently, $5 \mu 1$ of PI was added to the samples (final concentration of $1 \mu \mathrm{g} / \mathrm{ml}$ ) and 10000 cells were immediately analyzed using a FACSCalibur flow cytometer (Becton-Dickinson). Results were calculated as a percentage of apoptotic cells. Cell proliferation detection was performed using a Cell Counting Kit- 8 assay. According to the manufacturer's instructions, cells were paved into 96-well plates with 4000 cells per well. Cell proliferation intensity was calculated as the OD450 absorbent value for each well.

Protein extraction and Western blot analysis. The cells transfected with different plasmids or siRNAs were harvested at the indicated time points and washed twice with precooled PBS. Then, an appropriate volume of lysis buffer (20 mM Tris $\mathrm{HCl}, \mathrm{pH} 7.4,150 \mathrm{mM} \mathrm{NaCl}, 1 \mathrm{mM}$ EDTA, $1 \mathrm{mM}$ EGTA, $1 \mathrm{mM}$ PMSF and $1 \%$ Triton X-100) was added, sufficiently suspended, and reacted for $30 \mathrm{~min}$ at $4^{\circ} \mathrm{C}$. After that, the supernatant was collected and assayed using the BCA protein quantification kit (Pierce, USA).

The same amounts of protein lysates were separated by SDS-PAGE gel and electroblotted into an NC membrane (Amersham Pharmacia, UK). Then the membrane was incubated in 5\% BSA solution, dissolved using TBS-T (Trisbuffered saline containing $0.1 \%$ Tween-20) for $2 \mathrm{~h}$ at room temperature. Subsequently, specific primary antibodies were used and incubated overnight at $4{ }^{\circ} \mathrm{C}$, and secondary antibodies were incubated for $2 \mathrm{~h}$ at room temperature in the dark. The membranes were washed twice using TBST and scanned using an Odyssey Infrared Imager (LI-COR Bioscience) system.

Wound healing assay. The wound healing assay was used to detect cellular mobility. In our experiments, transfected cells were paved into 6-well plates and the cell layer was scratched using yellow tips. The scratched cells were washed twice with medium and cultured at $37^{\circ} \mathrm{C}$. The wounds were photographed at indicated time points $(0,36 \mathrm{~h})$ using phase contrast microscopy to monitor the wound healing process. 


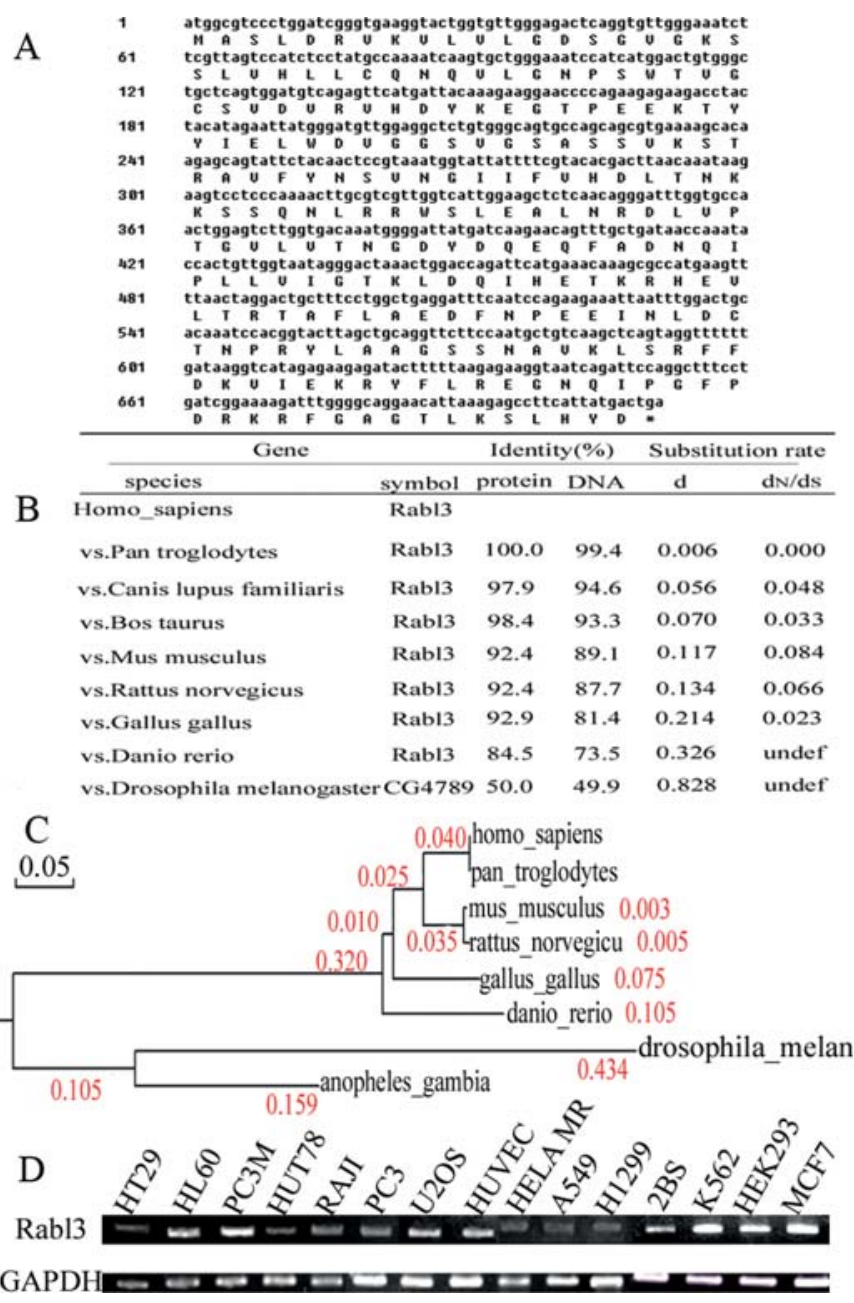

Figure 1. The novel Rabl3 gene. (A) Nucleotide sequences and encoded amino acid sequences of human gene Rabl3. (B) Sequence alignments of the Rabl3 gene. The homology alignment results were indicated using identity degrees. (C) Phylogenetic analysis of Rabl3. (DI) The expression profile of the Rabl3 gene analyzed using RT-PCR in various kinds of human cell lines originated from normal or cancer tissues.

Cellular detachment assay. The cellular detachment assay was performed to assess cellular adhesion ability. Transfected cells were paved into 6-well plates and cultured for $36 \mathrm{~h}$. Cells were then treated with $0.005 \%$ trypsin in $\mathrm{Mg}^{2+} / \mathrm{Ca}^{2+}$ free PBS. The trypsinized cells were photographed at indicated time points $(0,25$ or $50 \mathrm{~min})$. Cellular adhesive strength was assayed by determining relative sensitivity to trypsin.

Statistical analysis. All the experiments mentioned above were repeated at least 3 times. The results shown in each figure were calculated as the arithmetic means \pm SD. Statistical analysis was performed using the T-test. $\mathrm{P}<0.05$ was considered as statistically significant.

\section{Results}

Identification and expression pattern of Rabl3. In the present study, the full-length coding region of Rabl3 was directly isolated and amplified from the HeLa cell cDNA library by PCR using the specific primers P1 and P2. A 711 bp complete cDNA product was obtained and found to encode 236 amino acids with the predicted molecular weight of 26.307 KD. The cloned ORF sequence of the Rabl3 gene and encoded amino acid sequence are shown in Fig. 1A.

Based on the basic sequence analysis, human Rabl3 is located on $3 q 13.3$, and is composed of 8 exons and 7 introns. Upstream of the putative ATG start codon there exists an in-frame stop codon. The translation products of the Rabl3 gene have the isoelectric point of 6.31. By searching the GeneBank database and aligning sequences among different eukaryotes, it was found that Rabl3 is highly conserved in Homo sapiens, Pan troglodytes, Mus musculus, Rattus norvegicu, Gallus gallus, Danio rerio, Drosophila melan and Anopheles gambia (Fig. 1B and C).

Furthermore, the RT-PCR analysis was performed to identify the expression level in different cell lines. As shown in Fig. 1D, RT-PCR results clearly revealed that human gene Rabl3 was expressed ubiquitously and abundantly in serial cell lines originating from normal tissues, as well as cancer tissues.

Effects of Rabl3 overexpression on cell motility in cancer cells. It is known that cell motility plays a key role in tumor cell development and metastasis. To further evaluate the role of Rabl3 in tumorigenesis, the effect of this gene on cell mobility and adhesion was studied using F-actin staining, and functionally assessed using the wound healing and cell detachment assays.

For the wound healing assay, cells were transfected with Rabl3 and control plasmid and scratch wounding was carried out using a yellow pipette tip. Phase contrast images of the wound healing process were photographed at the indicated times. As shown in Fig. 2A, identical wound widths healed at different speeds in the different experimental groups. Human breast cancer cells overexpressed with Rabl3 exhibited strong healing activity. In contrast, control cells exhibited relatively slow healing activity towards the middle of the wound. This indicated that Rabl3 was crucial for promoting cell migration and suggested that it had an important role to play in the mobility and metastasis of tumor cells.

Furthermore, using the cell detachment assay, the results showed clearly that cells overexpressing Rabl3 demonstrated strong adhesion abilities. They were more resistant to trypsin, as compared with the negative controls (Fig. 2B and C). This finding further demonstrated the key role played by Rabl3 in the regulation of cell motility.

In addition, F-actin staining clearly revealed differences in morphological changes between Rabl3 overexpressed MDA-MB-231 and control cells (Fig. 2D). F-actin in Rabl3 transfected cells exhibited stronger staining with a cortical distribution, as compared with negative control cells. This finding also potentially supports the positive effects of Rabl3 on cell motility.

Effects of silencing gene Rabl3 on cell viability, motility and apoptosis in MDA-MB-231 cells. Since detection of functional changes in Rabl3 downregulated cells was considered necessary for our physiological studies, siRNA against Rabl3 was designed, synthesized and then used to silence the expression of Rabl3 in cancer cells. Non-silencing 


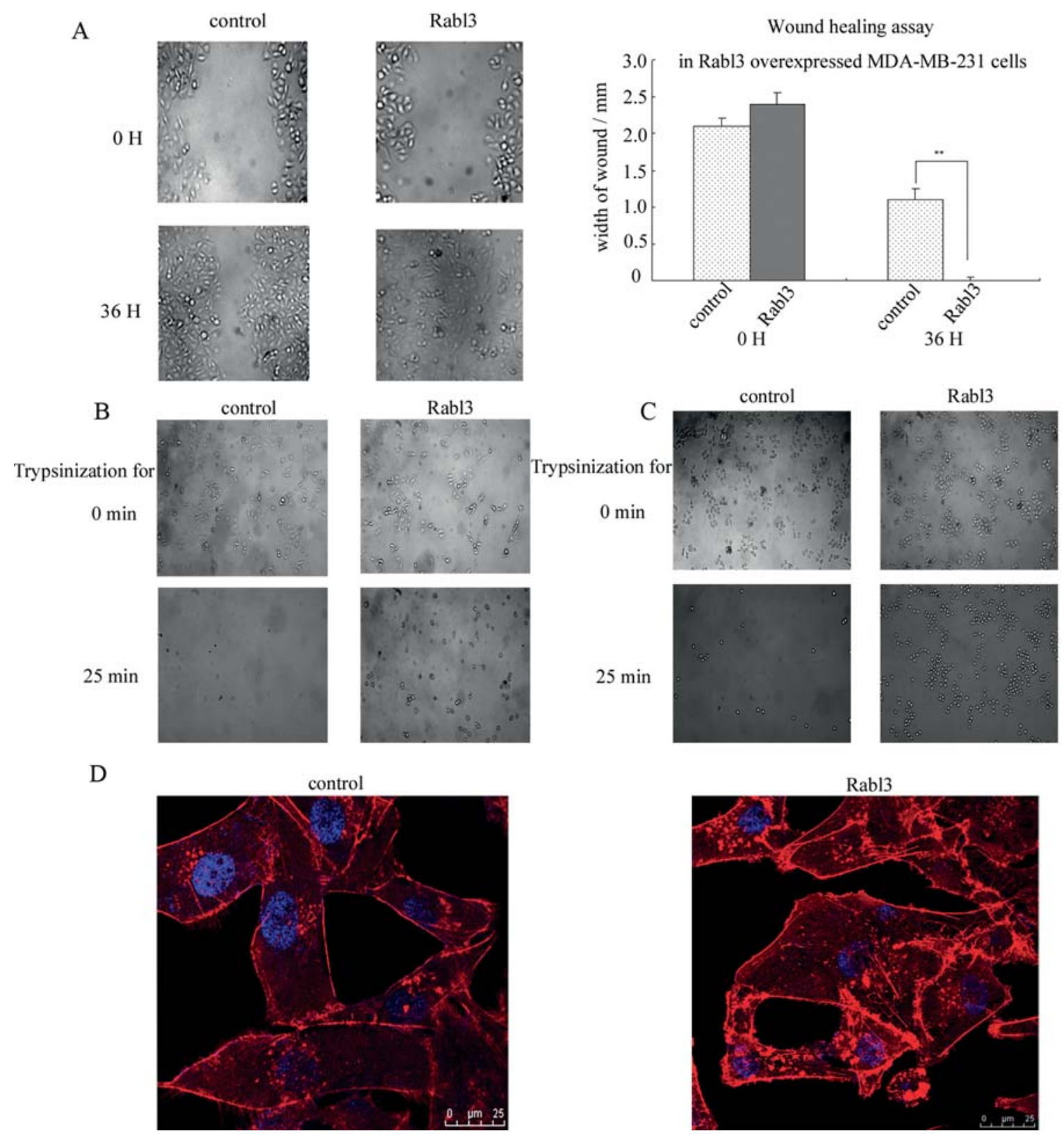

Figure 2. Detection of cellular motility changes in Rabl3 overexpressed cells. (A) Wound healing assay in Rabl3 overexpressed MDA-MB-231 cells. Phase contrast photographs showed that overexpression of Rabl3 enhanced the mobility of MDA-MB-231 cells at $36 \mathrm{~h}$ after transfection. The wound width can be used as the index reflecting the ability of cell migration between different experiment teams. (B) Cell detachment assay in Rabl3 overexpressed MDA-MB-231 cells. Cells were trypsinized using $0.005 \%$ trypsin in $\mathrm{Mg}^{2+} / \mathrm{Ca}^{2+}$ free PBS at $36 \mathrm{~h}$ after transfection. Phase contrast photographs were taken at the indicated time points after trypsinization. The density of adherent cells remaining in the plate represents adherent ability in different experimental groups. (C) Cell detachment assay in Rabl3 overexpressed HeLa cells. (D) F-actin staining with Phalloidin analyzed by confocal microscopy (Olympus, Tokyo, Japan). The pictures revealed that the Rabl3 overexpressed MDA-MB-231 cells exhibited a stronger signal of F-actin with cortical distribution.

siRNA termed 'si-non-RNA' was also used at the same time as the negative control.

RT-PCR and Western blot analysis confirmed that the Rabl3 specific si-RNA was efficient in blocking both the Rabl3 transcriptional and expressional procedures for 24 to $48 \mathrm{~h}$ after electroporation. In contrast, si-non-RNA had no effect on the Rabl3 expression (Fig. 3A).

To confirm that downregulation of Rabl3 could inhibit cell proliferation and initiate apoptosis, the CCK- 8 assay and Annexin V-PI staining were performed. Compared with the previous results obtained using Rabl3 overexpressed cells, the OD 450 value for Rabl3 downregulated cells was signi- ficantly lower $(\mathrm{P}<0.05)$ than that for the si-non-negative control group (data not shown). Next, we evaluated whether Rabl3 downregulation could restore or even enhance PCD condition. As shown in Fig. 3B, the findings from phosphatidylserine staining strongly supported this hypothesis, in that the fraction of apoptotic cells in si-Rabl3 transfected cells was obviously higher than in si-non cells. These results provided more evidence that Rabl3 functions as a key growth stimulator during tumor cell proliferation.

In addition, the relative migration abilities of si-Rabl3 and si-non transfected cells were compared. The results clearly demonstrated that a lack of Rabl3 expression caused 


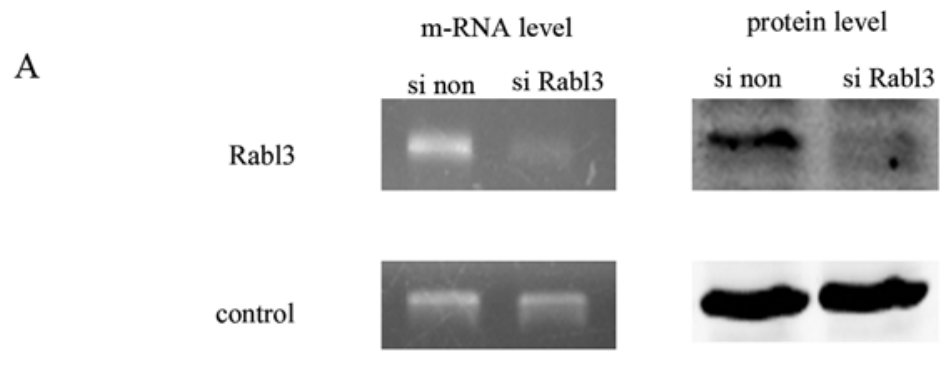

B
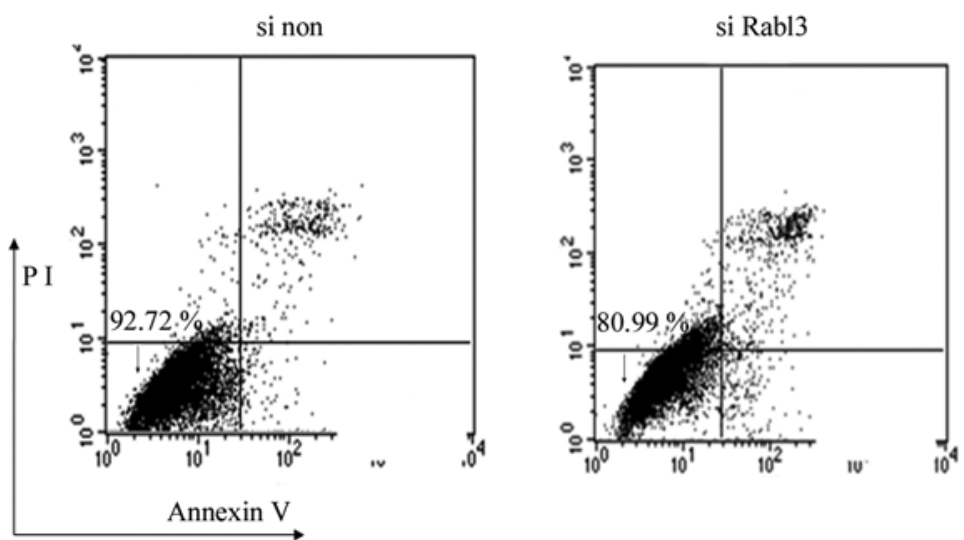

Detection of phosphatidylserine externalization
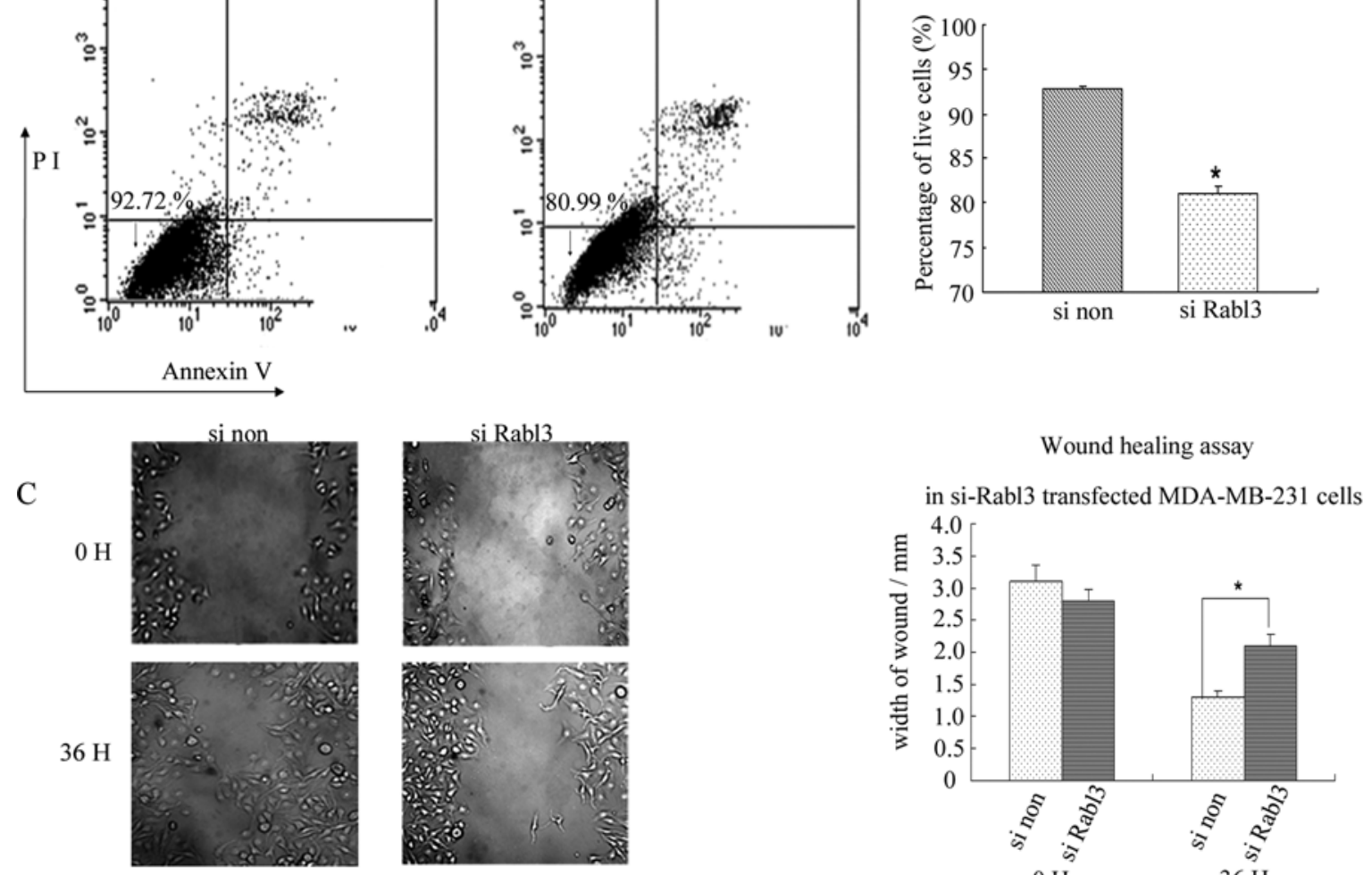

Figure 3. Detection of cellular viability and motility changes in Rabl3 siRNA transfected cancer cell lines. (A) Identification of siRNA against Rabl3 both in mRNA level and protein level. Cells were harvested at $36 \mathrm{~h}$ after transfection. (B) Detection of PS externalization in non-silencing and siRabl3 transfected MDA-MB-231 cells. Cells were harvested at $48 \mathrm{~h}$ after transfection. The percentages of Annexin V or PI single positive and Annexin V/PI double positive cells were calculated as the apoptotic intensity in different cells. The results were further quantified as the percentage of live cells shown in the diagram below. (C) Wound healing assay in siRNA transfected MDA-MB-231 cells. The width of the wound represents the wound healing ability in different experimental groups.

significant downregulation of migration activity in MDAMB-231 cells. The wound healing ability of these cells appeared lower than that of negative controls (Fig. 3C). Therefore, these data suggested that Rabl3 may be involved in both cell survival and migration.

Evaluation of FAK phosphorylation in Rabl3 overexpressed cancer cells. It is widely accepted that complicated regulation is involved in tumorigenesis and that there is a loss of the tight control on cell growth or apoptosis. To investigate how Rabl3 modulates cellular proliferation and migration processes, we screened and analyzed various signal pathways. We found a high phosphorylation level of FAK in Rabl3 overexpressed MDA-MB-231 cells. As is known, FAK is a non-receptor tyrosine kinases (NRPTK) which is localized in focal adhesions, multi-molecular structures involved in connections between the cytoskeleton and extracellular matrix (12). FAK is also a potential candidate signaling molecule. It is capable of receiving and integrating multiple signals transduced from integrin and regulating diverse signaling pathways downstream of FAK (13). Therefore, FAK mediates fundamental effects associated with complicated biological process, such as cell proliferation, migration and adhesion (14). In particular, for many kinds of cancers, FAK is a key signaling trigger which has close connection with cancer cell invasion and metastasis (15). To date, five phosphorylation sites have been identified on the FAK molecule, including Tyr397/577/861/925. Phosphorylation of these sites activates FAK into its fully functional state (16). In particular, the phosphorylation sites of Tyr 


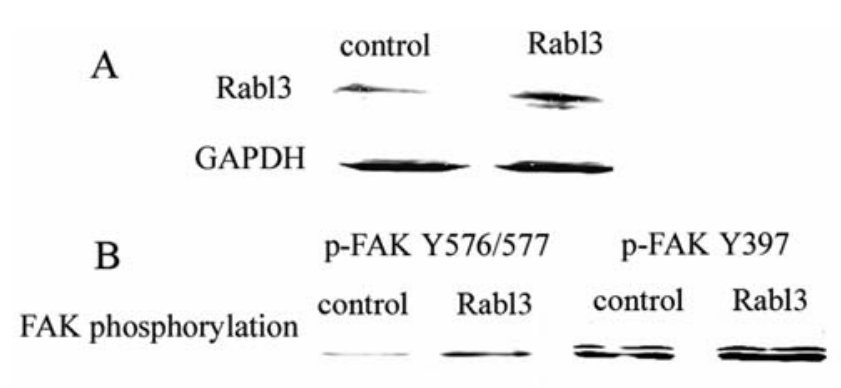

Total FAK

$\mathrm{C}$
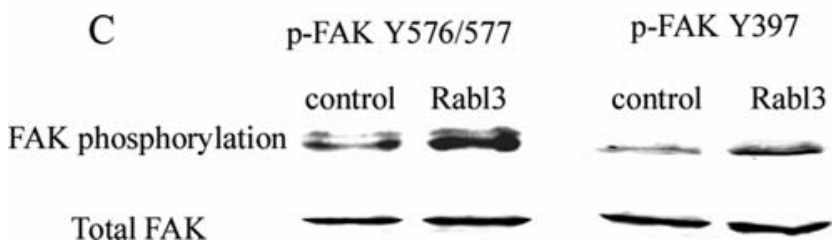

Figure 4. Rabl3 overexpression was accompanied by FAK phosphorylation. (A) Rabl3 overexpression identification in HeLa cells using anti-Rabl3 specific antibody at $36 \mathrm{~h}$ after transfection. (B) Rabl3 overexpression induced the increased FAK phosphorylation at the sites of Y397/ 576/577 in HeLa cells at $36 \mathrm{~h}$ after transfection. T-FAK was also detected as the internal control. (C) Western blot analysis of FAK phosphorylation in MDA-MB-231 cells.

576/577 endow the molecule with the strongest kinase activity.

Using Western blot analysis, we found that after efficient Rabl3 overexpression (Fig. 4A), the phosphorylation level of FAK Y397/576/577 was clearly higher than the level in empty vector transfected control cells. At the same time, the level of total FAK was found to be constant (Fig. 4B and C). This finding indicated that Rabl3 could strengthen FAK phosphorylation and then activate FAK, thus displaying the ability to activate proliferation and motility functions in cancer cells.

Sensitization of MDA-MB-231 cells to paclitaxel by inhibition of the Rabl3 gene. Over the years, there has been increasingly extensive use of the potent chemotherapeutic agent, paclitaxel. This agent is the first member of the taxel family (17) and is effective in treating certain types of cancer (18). Paclitaxel has been shown to stabilize microtube polymers, leading to mitotic arrest and apoptosis (19). However, drugresistance is a major but unsolved problem which limits the clinical efficacy of paclitaxel in approximately $50 \%$ of patients with breast cancers or other solid tumor types (20). In the case of patients undergoing long-term treatment with paclitaxel, the occurrence of drug-resistance is the primary obstacle to successful therapy (21). The precise mechanisms by which paclitaxel exerts its cytotoxic effects in tumor cells and the basic mechanism causing resistance to the drugs are still under wide discussion.

Our functional research related to Rabl3 revealed important influences on paclitaxel resistance in human breast cancer MDA-MB-231 cell lines. First of all, we tested paclitaxel sensitivity in MDA-MB-231 cells. As shown in Fig. 5A, paclitaxel cytotoxicity against MDA-MB-231 cells was concentration dependent. Photographs clearly showed that cells exhibited evidence of extensive cytoplasm vacuolization, blebbing and increased numbers of apoptotic cells detached from the cultural matrix after paclitaxel treatment. These findings indicated that MDA-MB-231 cells had high sensitivity to paclitaxel. Annexin V-PI staining clearly revealed that in overexpressed Rabl3 cells, the fraction of apoptotic cells induced by $7 \mu \mathrm{mol} / 1$ paclitaxel was relatively low at $24 \mathrm{~h}$ after the agent administration. However, we found that transfecting MDA-MB-231 cells with siRNA against Rabl3 resulted in the induction of significant numbers of apoptotic cells compared with the negative control, using paclitaxel at a concentration of $7 \mu \mathrm{mol} / \mathrm{l}$ (Fig. 5B and C). These changes in the proportion of apoptotic cells induced by paclitaxel, due to manipulation of the novel Rabl3 gene, indicate that there could be potential clinical benefits in targeting this gene.

In summary, the initial findings of our studies suggest that Rabl3 plays an active role in the generation of paclitaxel resistance. We demonstrated that silencing this gene can substantially restore, or even reverse, tumor cell resistance to paclitaxel.

\section{Discussion}

In the present study we investigated a novel human gene, Rabl3, which is highly conserved in a range of animal species. This gene is a member of the Rab subfamily. Rabs are mostly noted for the key roles that they play in regulating the formation of the cytoskeleton and dynamic transportation. It is clear that Rabs, although not usually considered oncogenic products, could profoundly affect the proliferation, survival and invasiveness of cancer cells. The Rab family loss or gain-of-function resulting from deletion or gene amplification could therefore promote cell transformation and/or enhance tumor cell motility.

The findings of the present study extend our understanding of the functional scope of this gene subfamily, and have contributed new evidence that Rabl3 functions as a positive regulator in connection with the processes of tumor cell viability and motility.

Revealing the signaling molecules involved in the Rabl3 oncological effects is a crucial area for further investigation. We have demonstrated for the first time that Rabl3 related signaling proteins play important roles in cellular proliferation and migration. Western blot analysis clearly showed that FAK phosphorylation levels were elevated as a result of Rabl3 overexpression at the sites of Y397/576/577. This finding implies a close relationship between Rabl3 and FAK. Since the FAK/Erk (Extracellular Regulatory Kinase) interaction is known to regulate many signaling transductions, and the functions of Erk in cell migration and survival are well documented $(22,23)$, we hypothesize that Rabl3 is a component in the FAK-Erk signaling pathway. Rabl3 have also been linked to cancer cell viability and migration, however, the underlying mechanism is unclear. Further studies need to be performed to reveal the exact biological behavior and interaction of FAK or other proteins. 
A
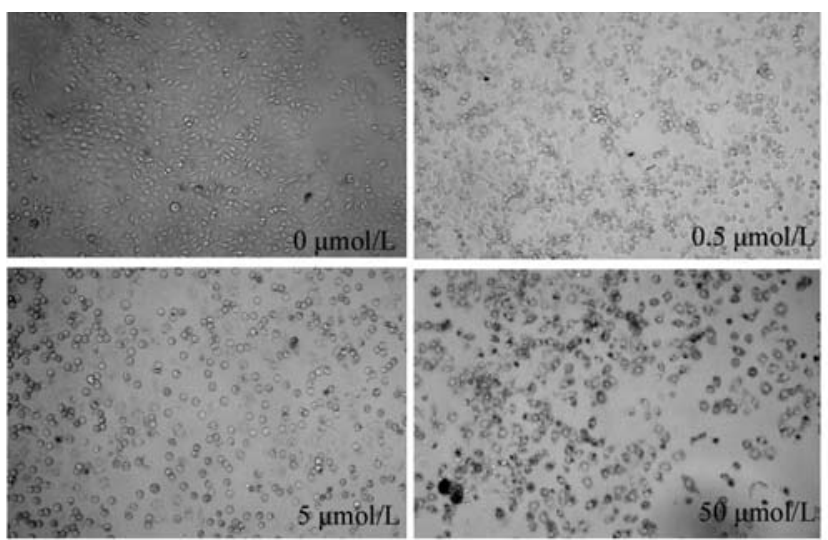
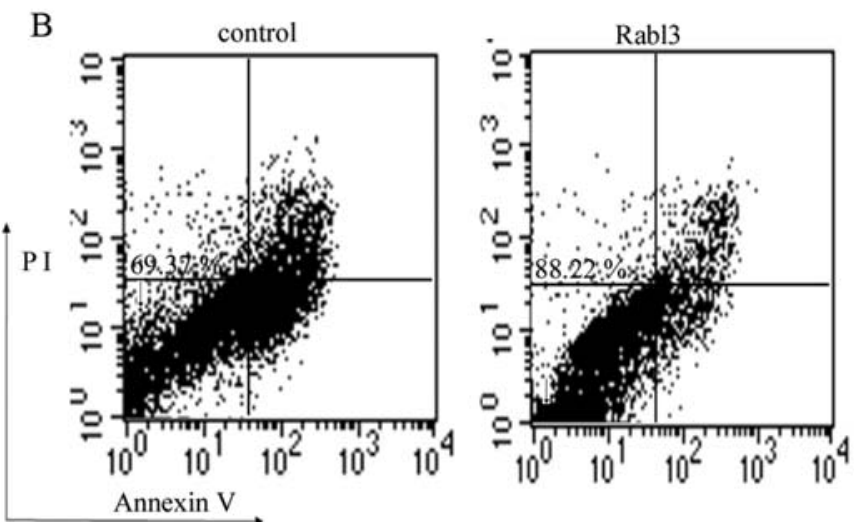

phosphatidylserine externalization after paclitaxel induction

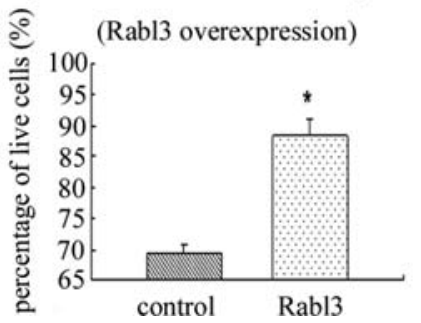

$\mathrm{C}$
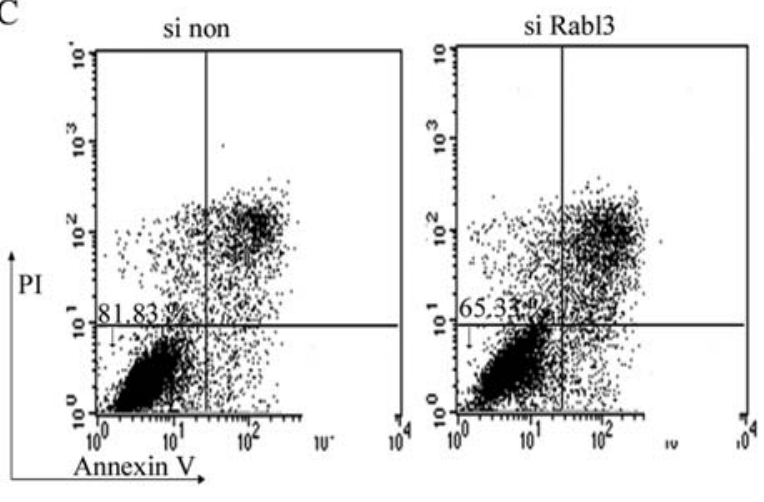

phosphatidylserine externalization after paclitaxel induction

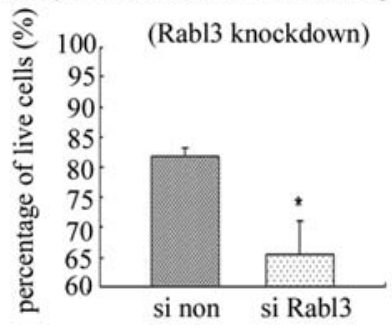

Figure 5 Sensitization of MDA-MB-231 cells to paclitaxel by inhibition of the Rabl3 gene. (A) The identification of paclitaxel sensitivity in MDA-MB-231 cells. The cytotoxicity induced by paclitaxel was both concentration and time-dependent in MDA-MB-231 cells. (B) Overexpression of Rabl3 induced paclitaxel resistance in MDA-MB-231 cells. The numbers of PI/Annexin V single positive and Annexin V/PI double positive cells were calculated as the measurement of apoptotic cells. The diagram shown below was utilized as the measurement of live cells within the total amounts of $1 \mathrm{x} 10^{4}$ detected cells. (C) Silencing of Rab13 expression increased paclitaxel induced apoptosis in MDA-MB-231 cells.

Anti-tumor agent resistance and non-sensitive treatment has become the most concentrated obstacles for cancer therapy; therefore, finding a new effective target of anti-drug resistance is very important. We have found that the novel human gene Rabl3 showed clear activity relating to paclitaxel sensitivity in breast cancer cells. Our results demonstrated that overexpression of Rabl3 could prevent tumor cells from undergoing paclitaxel-induced apoptosis, and as a consequence elevate resistance to paclitaxel. Silencing of Rabl3 expression could increase paclitaxel induced apoptosis in MDA-MB-231 cells. Given the importance of RAB GTPases in regulating many cellular functions, it is not surprising that altered expression or mutation of Rab proteins and their interacting partners may cause human diseases. However, as different kinds of PCD induced by paclitaxel can be triggered through a range of different mechanisms (24-27), the exact mechanism underlying Rabl3 mediated paclitaxel resistance remains unknown. Further studies with regard to this problem are needed.

The studies represent an important role of Rabl3 in cancer treatment. Based on recent research, substantial evidence indicated that some RAB gene members might be useful for prognostic and/or diagnostic markers in cancer therapy practice. Progress in this area is likely to improve our understanding of not only the role of RAB GTPase in the biology of cancer but also the essential role of cellular trafficking in the maintenance of a number of normal physiological processes. As the underlying mechanism of Rabmediated tumorigenesis comes to light, it is certain that Rab protein and their interaction molecules will represent a novel therapeutic target.

In conclusion, we have demonstrated that Rabl3 can enhance cell proliferation and migration activity, and inhibit 
apoptosis in human cancer cell lines exogenously. We also showed that silencing the Rabl3 gene can generate wholly opposite changes in cell activity. Moreover, we found that the biological functions of Rabl3 are largely connected with FAK phosphorylation at the sites of Y397576/577. Importantly, our findings have potential clinical implications. Loss of Rabl3 expression will cause an elevated sensitivity to paclitaxel in breast cancer cells, therefore, implicating Rabl3 might also be new potential targets for therapeutic strategies against breast cancer in viability and metastasis.

\section{Acknowledgements}

This study was supported by grants from the National Natural Science Foundation of China (30471581 and 30571702) and the National High Biotechnology Development Program of China (2002BA711A01-09). We sincerely appreciate the help and instructions of Professor Dalong Ma during our research.

\section{References}

1. Yang H, Sasaki T, Minoshima S and Shimizu N: Identification of three novel proteins (SGSM1, 2, 3) which modulate small G protein (RAP and RAB)-mediated signaling pathway. Genomics 90: 249-260, 2007.

2. Ramos FP, Araripe JR, Ürményi TP, Silva O, Silva NLC, Fontes CFL, Silveira JF and Rondinelli E: Characterization of RAB-like4, the first identified RAB-like protein from Trypanosoma cruzi with GTPase activity. Biochem Biophys Res Commun 333: 808-817, 2005.

3. Hagiwara M, Shirai Y, Nomura R, Sasaki M, Kobayashi K, Tadokoro $\mathrm{T}$ and YamamotoY: Caveolin-1 activates Rab5 and enhances endocytosis through direct interaction. Biochem Biophys Res Commun 378: 73-78, 2009.

4. Deneka M, Neeft $M$ and Van der Sluijs P: Regulation of membrane transport by Rab GTPases. Crit Rev Biochem Mol Biol 38: 121-142, 2003.

5. Dong $\mathrm{C}$ and Wu G: Regulation of anterograde transport of adrenergic and angiotensin II receptors by Rab2 and Rab6 GTPases. Cell Signal 19: 2388-2399, 2007.

6. Bizimungu $\mathrm{C}$ and Vandenbo $\mathrm{M}$ : At least two regions of the oncoprotein Tre2 are involved in its lack of GAP activity. Biochem Biophys Res Commun 335: 883-890, 2005.

7. Shan J, Mason JM, Yuan LM, Barcia M, Calabro DP, Budman D, Vinciguerra V and Xu HP: Rab6c, a new member of the Rab gene family, is involved in drug resistance in MCF7/AdrR cells. Gene 257: 67-75, 2000

8. Weaver V and Werb Z: G'rab'bing the microenvironment for invasion. Dev Cell 13: 462-463, 2007.

9. Caswell PT, Spence HJ, Parsons M,White DP, Clark K, Cheng KW, Mills GB, Humphries MJ, MessentA J, Anderson KI, McCaffrey MW, Ozanne BW and Norman JC: Rab25 Associates with a5b1 integrin to promote invasive migration in 3D microenvironments. Dev Cell 13: 496-510, 2007.

10. Cheng KW, Lu YL and Mills GB: Assay of Rab25 function in ovarian and breast cancers. Methods Enzymol 403: 202-215, 2005 .
11. Amillet JM, Ferbus D, Real F X, Antony C, Muleris M, Gress TM and Goubin G: Characterization of human Rab20 overexpressed in exocrine pancreatic carcinoma. Human Pathol 37: 256-263, 2006.

12. Tito NN, Robledo T and Salazar EP: Arachidonic acid promotes FAK activation and migration in MDA-MB-231 breast cancer cells. Exp Cell Res 314: 3340-3355, 2008

13. Fong YC, Liu SC, Huang CY, Li TM, Hsu SF, Kao ST, Tsai FJ, Chen WC, Chen CY and Tang CH: Osteopontin increases lung cancer cells migration via activation of the $\alpha v ß 3$ integrin/FAK/ Akt and NF- $\kappa \mathrm{B}$-dependent pathway. J Lung Cancer 64: 263$270,2008$.

14. Gilmore AP and Romer LH: Inhibition of focal adhesion kinase (FAK) signaling in focal adhesions decreases cell motility and proliferation. Mol Biol Cell 7: 1209-1124, 1996.

15. Lin AH, Eliceiri BP and Levin EG: FAK mediates the inhibition of glioma cell migration by truncated $24 \mathrm{kDa}$ FGF-2. Biochem Biophys Res Commun 382: 503-507, 2009.

16. Baillat G, Siret C, Delamarre E and Luis J: Early adhesion induces interaction of FAK and Fyn in lipid domains and activates raft-dependent Akt signaling in SW480 colon cancer cells. Biochim Biophys Acta 1783: 2323-2331, 2008.

17. Marupudi NI, Han JE, Li KW, Renard VM, Tyler BM and Brem H: Paclitaxel: a review of adverse toxicities and novel delivery strategies. Expert Opinion Drug Saf 6: 609-621, 2007.

18. Chen TS, Wang XP, Sun L, Wang LX, Xing D and Mok M: Taxol induces caspase-independent cytoplasmic vacuolization and cell death through endoplasmic reticulum (ER) swelling in ASTC-a-1 cells. Cancer Lett 270: 164-172, 2008.

19. Schiff PB, Fant J and Horwitz SB: Promotion of microtubule assembly in vitro by taxol. Nature 277: 665-667, 1979.

20. Ahmed AA, Mills AD, Ibrahim AEK, Temple J, Blenkiron C, Vias M, Massie CE, Iyer NG, McGeoch A, Crawford R, Nicke B, Downward J, Swanton C, Bell SD, Earl HM, Laskey RA, Caldas $\mathrm{C}$ and Brenton JD: The extracellular matrix protein TGFBI induces microtubule stabilization and sensitizes ovarian cancers to paclitaxel. Cancer Cell 12: 514-527, 2007.

21. Michele MD, Corte AD, Cicchillitti L, Boccio PD, Urbani A, Ferlini C, Scambia G, Donati MB and Rotilio D: A proteomic approach to paclitaxel chemoresistance in ovarian cancer cell lines. Biochim Biophys Acta 1794: 225-236, 2009.

22. Yu CF, Han WL, Shi TP, Lv BF, He QH, Zhang YF, Li T, Zhang YM, Song QS, Wang L and Ma DL: PTPIP51, a novel 14-3-3 binding protein, regulates cell morphology and motility via Raf-ERK pathway. Cell Signal 20: 2208-2220, 2008.

23. Luchetti F, Betti M, Canonico B, Arcangeletti M, Ferri P, Galli F and Papa S: ERK MAPK activation mediates the anti-apoptotic signaling of melatonin in UVB-stressed U937 cells. Free Radic Biol Med 46: 339-351, 2009.

24. Huisman C, Ferreira CG, Broker LE, Rodriguez JA, Smit EF, Postmus PE, Kruyt FAE and Giaccone G: Paclitaxel triggers cell death primarily via caspase independent routes in the nonsmall cell lung cancer cell line NCI-H4601. Clin Cancer Res 596: 596-606, 2002.

25. Selimovic D, Hassan M, Haikel Y and Hengge UR: Taxolinduced mitochondrial stress in melanoma cells is mediated by activation of c-Jun N-terminal kinase (JNK) and p38 pathways via uncoupling protein 2. Cell Signal 20: 311-322, 2008.

26. Hsiu K, Lue KH, Liao HH, Lin KL and Chung JG: Induction of caspase-3-dependent apoptosis in human leukemia HL-60 cells by paclitaxel. Clin Chim Acta 357: 65-73, 2005.

27. Berndtsson M, Konishi Y, Bonni A, Hagg M, Shoshan M, Linder S and Havelka AM: Phosphorylation of BAD at Ser-128 during mitosis and paclitaxel-induced apoptosis. FEBS Lett 579: 3090-3094, 2005. 